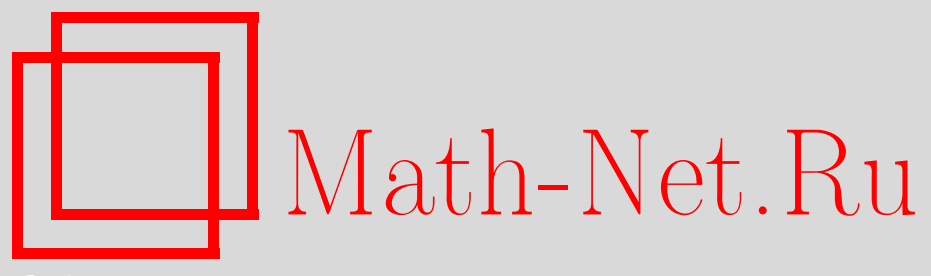

М. В. Житлухин, О совместном распределении $\sup \left(B_{s}-\right.$ $\mu s)$ и $\inf \left(B_{s}-\nu s\right)$ для броуновского движения $B_{s}, У M H$, 2008, том 63, выпуск 6, 163-164

DOI: https://doi.org/10.4213/rm9255

Использование Общероссийского математического портала Math-Net.Ru подразумевает, что вы прочитали и согласны с пользовательским соглашением http://www . mathnet.ru/rus/agreement

Параметры загрузки:

IP: 54.174 .149 .18

26 апреля 2023 г., 10:35:31

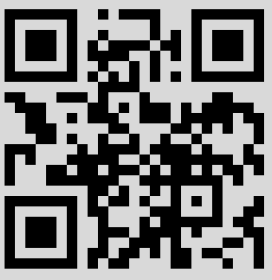


О совместном распределении $\sup \left(B_{s}-\mu s\right)$ и $\inf \left(B_{s}-\nu s\right)$ для броуновского движения $B_{s}$

\section{М. В. Житлухин}

1. Пусть $B=\left(B_{s}\right)_{s \geqslant 0}$ - стандартное броуновское движение, $\mu$ и $\nu$ - произвольные действительные числа и $a>0>b$. Определим вероятности

$$
\begin{aligned}
& F_{t}(a, b, y)=\mathrm{P}\left\{\sup _{s \leqslant t}\left(B_{s}-\mu s\right)<a, \inf _{s \leqslant t}\left(B_{s}-\nu s\right)>b \mid B_{t}=y\right\}, \\
& G_{t}(a, b)=\mathrm{P}\left\{\sup _{s \leqslant t}\left(B_{s}-\mu s\right)<a, \inf _{s \leqslant t}\left(B_{s}-\nu s\right)>b\right\} .
\end{aligned}
$$

Основной результат настоящей работы состоит в следующем.

Теорема 1. Для любого $t$ в случае $\mu \geqslant \nu u t<(a-b) /(\nu-\mu)$ в случае $\mu<\nu$ u любого $y \in[b+\nu t, a+\mu t]$ вероятность $F_{t}(a, b, y)$ определяется формулой

$$
\begin{aligned}
F_{t}(a, b, y)= & \sum_{k=-\infty}^{+\infty} \exp \left\{-\frac{2 k^{2}(a-b)}{t}(a-b+(\mu-\nu) t)-2 k\left(a \nu-b \mu-\frac{(a-b) y}{t}\right)\right\} \\
& -\sum_{k=-\infty}^{+\infty} \exp \left\{-\frac{2}{t}(k(a-b)+a)(k(a-b+(\mu-\nu) t)+a+\mu t-y)\right\}
\end{aligned}
$$

Теорема 2. Для любого $t$ в случае $\mu \geqslant \nu u t<(a-b) /(\nu-\mu)$ в случае $\mu<\nu$ вероятность $G_{t}(a, b)$ определяется формулой

$$
\begin{aligned}
G_{t}(a, b)= & \sum_{k=-\infty}^{+\infty} e^{-2 k^{2}(a-b)(\mu-\nu)-2 k(a \nu-b \mu)} \frac{1}{\sqrt{2 \pi t}} \int_{b+\nu t}^{a+\mu t} e^{-(x-2 k(a-b))^{2} /(2 t)} d t \\
& -\sum_{k=-\infty}^{+\infty} e^{-2(k(a-b)+a)(k(\mu-\nu)+\mu)} \frac{1}{\sqrt{2 \pi t}} \int_{b+\nu t}^{a+\mu t} e^{-(x-2(k+1) a+2 k b)^{2} /(2 t)} d t .
\end{aligned}
$$

Отметим, что вопрос об отыскании интересующих нас вероятностей, а также преобразования Лапласа момента $\tau=\inf \left\{s \geqslant 0: B_{s}=a+\mu s\right.$ или $\left.B_{s}=b+\nu s\right\}$, где $\inf \varnothing=\infty$, исследовался во многих работах и разными методами (распределение $\tau$ связано с $G_{t}(a, b)$ соотношением $\left.G_{t}(a, b)=\mathrm{P}\{\tau>t\}\right)$.

Так, в [1] для отыскания вероятности $G_{t}(a, b)$ использовался метод дифференциальных уравнений, что приводит к достаточно громоздким вычислениям. В работе [2] в случае $\mu \geqslant \nu$ для отыскания преобразования Лапласа момента $\tau$ использовался "мартингальный" метод. Но, как отмечает автор этой работы, его метод не работает в случае $\mu<\nu$.

2. Доказательство формул (2) и (3) основывается на следующей теореме.

Teopema 3. Пусть $a>0>b, \mu>\nu$. Тогдa

$$
\begin{aligned}
\mathrm{P}\left\{\sup _{s \geqslant 0}\left(B_{s}-\mu s\right)<a, \inf _{s \geqslant 0}\left(B_{s}-\nu s\right)>b\right\} \\
\quad=\sum_{k=-\infty}^{+\infty} e^{-2 k^{2}(a-b)(\mu-\nu)+2 k(a \nu-b \mu)}-\sum_{k=-\infty}^{+\infty} e^{-2(k(\mu-\nu)+\mu)(k(a-b)+a)} .
\end{aligned}
$$


Идея доказательства теоремы 3 сходна с идеей [2] и основана на рассмотрении мартингала $M_{t}^{\lambda}=e^{\lambda B_{t}-t \lambda^{2} / 2}, t \geqslant 0$. Используя теорему об остановке (см. [3; гл. II, §3]), можно показать, что для $\lambda \notin(2 \nu, 2 \mu)$ выполнено равенство $\mathrm{E} M_{\tau}^{\lambda} I(\tau<\infty)=1$, эквивалентное равенству

$$
\begin{aligned}
& e^{\lambda a} \mathrm{E} e^{\tau\left(\lambda \mu-\lambda^{2} / 2\right)} I\left(B_{\tau}=a+\mu \tau, \tau<\infty\right) \\
& \quad+e^{\lambda b} \mathrm{E}^{\tau\left(\lambda \nu-\lambda^{2} / 2\right)} I\left(B_{\tau}=b+\nu \tau, \tau<\infty\right)=1 .
\end{aligned}
$$

Выбирая специальным образом последовательность $\left\{\lambda_{k}\right\}_{k \geqslant 1}$, можно получить рекуррентные соотношения для $x_{k}=\mathrm{E} e^{\tau\left(\lambda_{k} \mu-\lambda_{k}^{2} / 2\right)} I\left(B_{\tau}=a+\mu \tau, \tau<\infty\right)$.

А именно, положим $\lambda_{2 k-1}=2(k-1)(\mu-\nu)+2 \mu, \lambda_{2 k}=2 \nu-\lambda_{2 k-1}=-2 k(\mu-\nu)$. Тогда, используя (4), получаем соотношение

$$
x_{2 k-1}=e^{-\lambda_{2 k-1} a}-e^{b\left(\lambda_{2 k-1}-\lambda_{2 k}\right)-\lambda_{2 k-1} a}+e^{(a-b)\left(\lambda_{2 k}-\lambda_{2 k-1}\right)} x_{2 k+1},
$$

из которого находим формулу для $x_{1}=\mathrm{E} I\left(B_{\tau}=a+\mu \tau, \tau<\infty\right)$ :

$$
x_{1}=\sum_{k=0}^{\infty} e^{-2(k(\mu-\nu)+\mu)(k(a-b)+a)}-\sum_{k=1}^{\infty} e^{-2 k^{2}(a-b)(\mu-\nu)+2 k(a \nu-b \mu)} .
$$

Аналогично находится формула для $\mathrm{E} I\left(B_{\tau}=b+\nu \tau, \tau<\infty\right)$. Отсюда легко получить утверждение теоремы, так как искомая вероятность равна $\mathrm{P}\{\tau=\infty\}$.

3. Для доказательства теоремы 1 воспользуемся тем, что $\operatorname{Law}\left(B_{s}\right)=\operatorname{Law}\left(s B_{1 / s}\right)$. Тогда (1) можно переписать так (для простоты ограничимся случаем $t=1$ ):

$$
F_{1}(a, b, y)=\mathrm{P}\left\{\sup _{s \leqslant 1}\left(s B_{1 / s}-\mu s\right) \leqslant a, \inf _{s \leqslant 1}\left(s B_{1 / s}-\nu s\right) \geqslant b \mid B_{1}=y\right\} .
$$

Сделав замену $u=1 / s$ и воспользовавшись марковским свойством броуновского движения, получаем, что

$$
F_{1}(a, b, y)=\mathrm{P}\left\{\sup _{u \geqslant 0}\left(B_{u}-a u\right) \leqslant a+\mu-y, \inf _{u \geqslant 0}\left(B_{u}-b u\right) \geqslant b+\nu-y\right\} .
$$

Применяя теперь теорему 3, получаем формулу (2).

Теорема 2 доказывается интегрированием формулы (2).

\section{Список литературы}

[1] M. Teunen, M. Goovaerts, Scand. Actuar. J., 2 (1994), 139-150. [2] L. Barba Escriba, Ann. Probab., 15:4 (1987), 1524-1526. [3] D. Revuz, M. Yor, Continuous martingales and Brownian motion, 3rd ed., Grundlehren Math. Wiss., 293, Springer-Verlag, Berlin, 1999.

M. В. Житлухин (M. V. Zhitlukhin)

Московский государственный университет им. М. В. Ломоносова

E-mail: zhitlukhin@gmail.com
Представлено Д. В. Трещёвым Принято редколлегией 27.10.2008 